\title{
Mechanism transition and strong temperature dependence of dislocation nucleation from grain boundaries: An accelerated molecular dynamics study
}

\author{
Jun-Ping Du, ${ }^{1,2}$ Yun-Jiang Wang, $, 3,{ }^{3, *}$ Yu-Chieh Lo, ${ }^{5}$ Liang Wan, ${ }^{2,6}$ and Shigenobu Ogata ${ }^{2,1, \dagger}$ \\ ${ }^{1}$ Center for Elements Strategy Initiative for Structural Materials (ESISM), Kyoto University, Sakyo, Kyoto 606-8501, Japan \\ ${ }^{2}$ Department of Mechanical Science and Bioengineering, Osaka University, Osaka 560-8531, Japan \\ ${ }^{3}$ State Key Laboratory of Nonlinear Mechanics, Institute of Mechanics, Chinese Academy of Sciences, Beijing 100190, China \\ ${ }^{4}$ School of Engineering Science, University of Chinese Academy of Sciences, Beijing 101408, China \\ ${ }^{5}$ Department of Materials Science and Engineering, National Chiao Tung University, 1001 University Road, Hsinchu City 300, Taiwan \\ ${ }^{6}$ Center for Advancing Materials Performance from the Nanoscale, State Key Laboratory for Mechanical Behavior of Materials, \\ Xi'an Jiaotong University, Xi'an 710049, China
}

(Received 24 May 2016; revised manuscript received 6 August 2016; published 14 September 2016)

\begin{abstract}
Accelerated molecular dynamics reveals a mechanism transition and strong temperature dependence of dislocation nucleation from grain boundaries (GBs) in $\mathrm{Cu}$. At stress levels up to $~ 90 \%$ of the ideal dislocationnucleation stress, atomic shuffling at the $E$ structural unit in a GB acts as a precursor to dislocation nucleation, and eventually a single dislocation is nucleated. At very high stress levels near the ideal dislocation-nucleation stress, a multiple dislocation is collectively nucleated. In these processes, the activation free energy and activation volume depend strongly on temperature. The strain-rate dependence of the critical nucleation stress is studied and the result shows that the mechanism transition from the shuffling-assisted dislocation-nucleation mechanism to the collective dislocation-nucleation mechanism occurs during the strain rate increasing from $10^{-4} \mathrm{~s}^{-1}$ to $10^{10} \mathrm{~s}^{-1}$.
\end{abstract}

DOI: 10.1103/PhysRevB.94.104110

\section{INTRODUCTION}

Dislocation nucleation from interfacial defects dominate plastic deformation of materials in a confined volume, which may have a limited number of plastic deformation carriers [14]. For example, plastic deformation of nanocrystalline metals, which exhibit high strength, is governed by a dislocationnucleation event from grain boundaries (GBs) [5]. In general, dislocation nucleation is an effective way to release strain energy in the locally stress-concentrated regions, such as GB during the dislocation transmission [6], the tip of cracks $[7,8]$, and triple lines in polycrystalline materials with strain incompatibilities [9].

Molecular dynamics (MD) is one of the best tools for studying the dislocation nucleation from GBs at atomiclevel resolution. Actually, the dislocation-nucleation-mediated deformation mechanism of nanocrystalline metals [10-12] has been captured using MD simulations, which can qualitatively explain experimental observations $[1,13]$. The strain rate in MD simulations $\left(\sim 10^{8} \mathrm{~s}^{-1}\right)$, however, differs considerably from typical experimental strain rates $\left(\sim 10^{-4} \mathrm{~s}^{-1}\right)$ because of the short time scale of MD simulations. Because dislocation nucleation is a thermally activated rate-controlling process at finite temperature and is usually rate sensitive, the temperature and strain-rate sensitivities should be carefully studied at realistic time scales $[14,15]$. Thus in this study we accelerate the dislocation-nucleation events using adaptive-boost MD (ABMD) $[16,17]$ and study the dislocation nucleation with atomic-level resolution at finite temperature, which offers opportunities for more comprehensive investigation of these processes arising from interfacial defects. Note that while the temperature and strain-rate dependences of dislocation nucle-

\footnotetext{
*yjwang@imech.ac.cn

†ogata@me.es.osaka-u.ac.jp
}

ation from surfaces have been studied using both atomistic modeling [18] and experiments [4], those of dislocation nucleation from GBs have not been studied yet. Hence, although the dislocation-nucleation mechanism has been often studied using typical MD simulation, the fundamental mechanisms are unfortunately not fully understood. For example, a MD simulation showed that the dislocation nucleation can be triggered by atomic shuffling with a stress-assisted free volume migration $[19,20]$ using a nanocrystalline model that consists of general GBs, but the other many MD simulations showed a collective nucleation manner without atomic shuffling [21-23]. One possibility of the absence of atomic shuffling in these MD simulations is that the diffusive processes of small activation volume, like the shuffling-assisted dislocation nucleation, are suppressed by the high strain rate of MD simulations, and the more displacive-stress-sensitive process, like the collective dislocation nucleation, is activated instead of the diffusive processes at such a high strain rate. On the other hand, since local atomic structure of the general GBs is more disordered with large free volume, a more diffusive mode is preferred in this case, and thus the shuffling may have been shown in former MD simulation.

Different atomic structures at GBs can generally lead to different dislocation-nucleation responses, and thus influence the overall mechanical performance of nanostructured materials having GBs, such as nanocrystalline materials, nanopillar, nanobeam, nanowire, and so on. To study dislocation nucleation from GBs, the $\Sigma 9\langle 110\rangle\{221\}$ symmetric tilt GB (STGB) in $\mathrm{Cu}$ was selected in this study for investigation. The structure of the $\Sigma 9\langle 110\rangle\{221\}$ STGB consists entirely of the $E$ structural unit, which contains a large free volume [24]. A critical role of the $E$ structural unit as the dislocation source at GBs $[22,25]$ has been suggested not only for special GBs such as the $\Sigma 9\langle 110\rangle\{221\}$ STGB GB but also for the general GBs, which also usually contain the $E$ structural unit [26]. The embedded-atom potential for $\mathrm{Cu}$ constructed by Mishin 


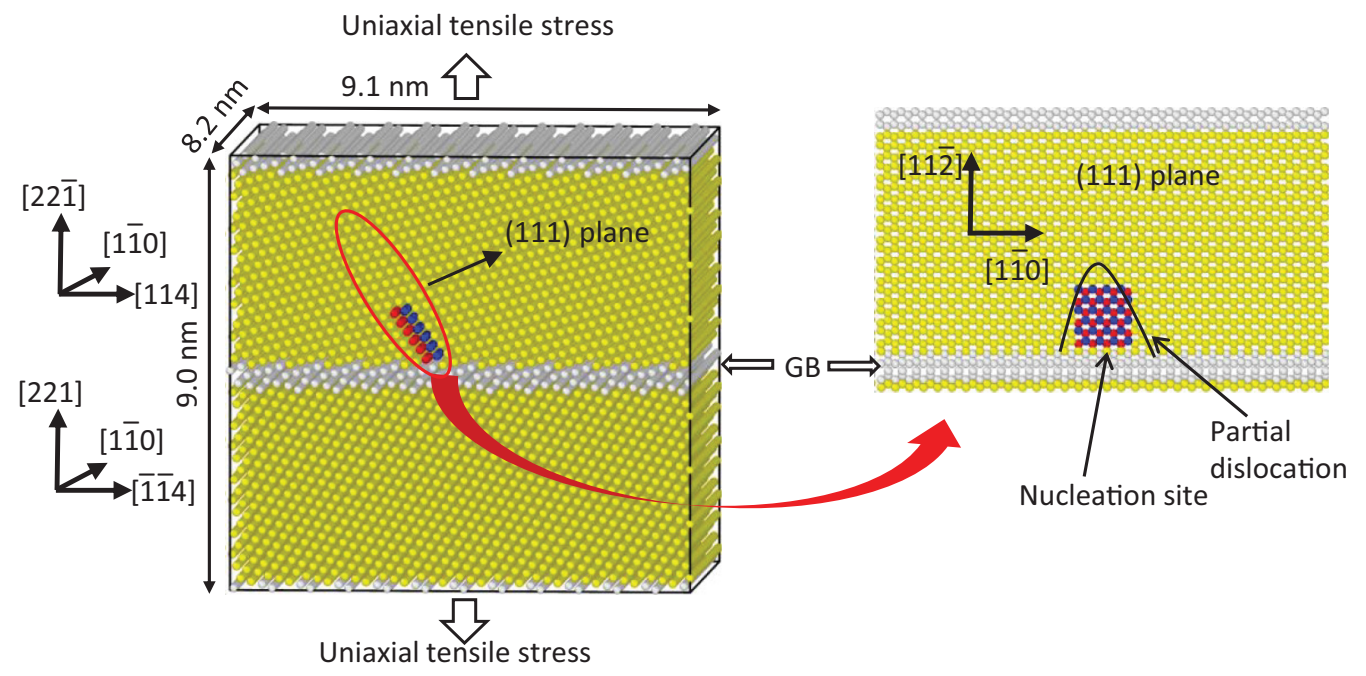

FIG. 1. Atomistic configuration of $\Sigma 9\langle 110\rangle\{221\}$ STGB of $\mathrm{Cu}$. Atoms in the GB region are white and other ones are the intragrain atoms. The GB atoms are recognized using common neighbor analysis [30] implemented in the software OvITO [31]. Blue and red atoms are the boosted atoms in the ABMD method. Definition of the nucleation site of the nucleated partial dislocation is also schematically shown in the right panel.

et al. [27], which has been well validated using experimental and first-principles data, is used to describe the interatomic interaction.

\section{MODEL AND SIMULATION METHOD}

\section{A. Model for ABMD and conventional MD}

The bicrystal model used in this work contains $55296 \mathrm{Cu}$ atoms with the crystallographic orientations as shown in Fig. 1. The model size is $9.1 \times 8.2 \times 9.0 \mathrm{~nm}^{3}$. Periodic boundary conditions (PBCs) are imposed in all three directions. Tensile stress was applied by a uniaxial constant strain along the $Z$ direction. The model sizes along the $X$ and $Y$ directions are adjusted according to the zero-pressure conditions.

\section{B. Free-end nudged elastic band method}

The free-end nudged elastic band (FENEB) method [28,29] has been widely used to calculate the activation energy of dislocation nucleation at $0 \mathrm{~K}$. Because of the large critical size of the nucleated dislocation at $0 \mathrm{~K}$, we use a model larger than that used in ABMD and conventional MD in the FENEB calculations. The model is $18 \times 16 \times 18 \mathrm{~nm}^{3}$ in size, which is large enough to eliminate the image effect of the PBC. The crystallographic orientations of the model are shown in Fig. 1. The spring constant in the FENEB method is set to be $0.1 \mathrm{eV}^{-2}$, and the calculation converges when the forces on the atoms are less than $0.002 \mathrm{eV} \AA^{-1}$.

\section{Adaptive-boost molecular dynamics method}

The ABMD method is used to evaluate the nucleation frequency under conditions of constant stress and temperature, at which regular MD is not available. A preconceived "important" event, which is dislocation nucleation from GB in this case, is accelerated in the ABMD method. This method adds a bias potential (boost potential) to the original potential
(Hamiltonian), and the bias potential induces a fictitious force on "boosted atoms." The boost potential is constructed as a function of a collective variable $(\mathrm{CV})$, and the $\mathrm{CV}$ is a function of the positions of boosted atoms. The boost potential is defined as

$$
\Delta V(\boldsymbol{A})=\left\{\begin{array}{cl}
k_{\mathrm{B}} T \ln \frac{\rho(\boldsymbol{A})}{\rho_{\text {cut }}}, & \rho(\boldsymbol{A}) \geqslant \rho_{\text {cut }} \\
0, & \rho(\boldsymbol{A})<\rho_{\text {cut }}
\end{array},\right.
$$

where $\rho(\boldsymbol{A})$ is a probability density of $\mathrm{CV} \boldsymbol{A}, k_{\mathrm{B}}$ is the Boltzmann constant, $T$ is the temperature, and $\rho_{\text {cut }}$ is the cutoff density. $\rho_{\text {cut }}$ is used to remove the poor statistic of $\rho(\boldsymbol{A})$ whose values are less than $\rho_{\text {cut }}$, so that the boost energy decreases to 0 smoothly at $\rho_{\text {cut }}$. In this study, $\rho_{\text {cut }}$ is set to be about $1.5 \%$ of the maximum of $\rho(\boldsymbol{A})$. The boost potential is self-adaptively constructed after each standard MD sampling of $10^{6}$ steps, which is referred to as one ABMD step in the paper. The time step for the ABMD simulation is set to be $1 \mathrm{fs}$. The ABMD method gives the nucleation frequency $\left(v_{i}\right)$ from one nucleation site, which is defined as the position of the "central atom" of the half dislocation loop (see Fig. 1). Here we assume that every atom which is equivalent to the central atom corresponds to an equivalent nucleation site. The total nucleation frequency $v(\sigma, T)$ at uniaxial tensile stress $\sigma$ and temperature $T$ is given by $v(\sigma, T)=N v_{i}$, where $N=1536$ is number of equivalent nucleation sites (see Supplemental Material [32]).

The choice of $\mathrm{CV}$ is important because it influences the reliability of ABMD. For dislocation nucleation from GBs, a relative displacement on both sides of the slip plane, which equals the magnitude of the Burgers vector of the partial dislocation, can characterize the nucleation process. Therefore, the relative displacement is a good $\mathrm{CV}$ to describe the nucleation pathway for dislocation nucleation. In this study, the one-dimensional $\mathrm{CV}$ is defined as $A=\boldsymbol{k} \cdot\left(\frac{\sum_{i}^{N_{1}} \boldsymbol{r}_{i}}{N_{1}}-\frac{\sum_{j}^{N_{2}} \boldsymbol{r}_{j}}{N_{2}}\right)$, where $N_{1}$ and $N_{2}$ are the numbers of boosted atoms (blue and red atoms in Fig. 1) of two adjoining (111) planes, 
which are used to construct the $\mathrm{CV}$, and $\boldsymbol{r}$ is the position vector of atoms. Thus, $\frac{\sum_{i}^{N_{1}} \boldsymbol{r}_{i}}{N_{1}}$ and $\frac{\sum_{j}^{N_{2}} \boldsymbol{r}_{j}}{N_{2}}$ are the centers of mass of the two groups of atoms (corresponding to the blue and the red atoms in Fig. 1, respectively). Further, $\boldsymbol{k}$ is the projection direction along the Burgers vector of a partial dislocation ([112] direction). The CV evolves according to the embedded-atom method potential and the self-adaptive boost potential. The GB atoms are near, but not included among, the boosted atoms because the displacement directions of GB atoms may differ from those of atoms outside the GB. The nucleation frequency is affected by the number of boosted atoms, $N_{\mathrm{b}}\left(=N_{1}+N_{2}\right)$, where $N_{1}$ and $N_{2}$ are the number of blue and red atoms in Fig. 1. A series of $N_{\mathrm{b}}$ is used to calculate the nucleation frequency for the shuffling-assisted single dislocation-nucleation mechanism at 2.5 and $2.7 \mathrm{GPa}$ at a temperature of $300 \mathrm{~K}$. The results are shown in Fig. 2. The dislocation-nucleation frequencies decrease with increasing $N_{\mathrm{b}}$. However, the frequency is insensitive to small $N_{\mathrm{b}}$. The nucleation frequency is affected by $N_{\mathrm{b}}$ because the bias potential tries to move the atoms simultaneously. Thus, the size of boosted atoms is approximately the largest size of dislocation nuclei that can appear in ABMD simulations. When the size of boosted atoms is much larger than the

(a)

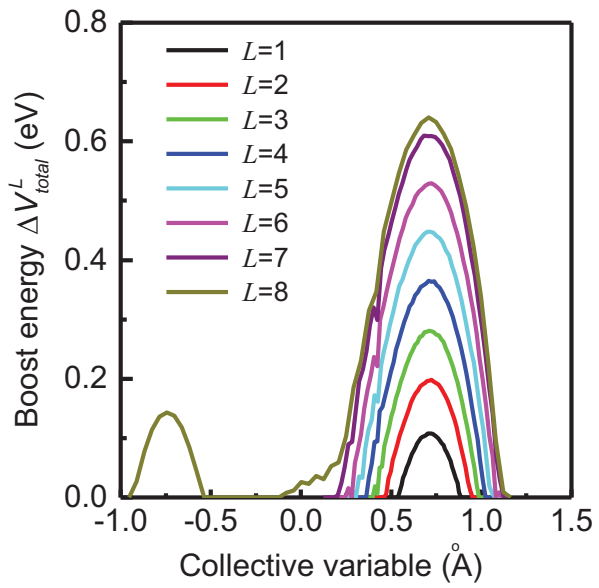

(c)

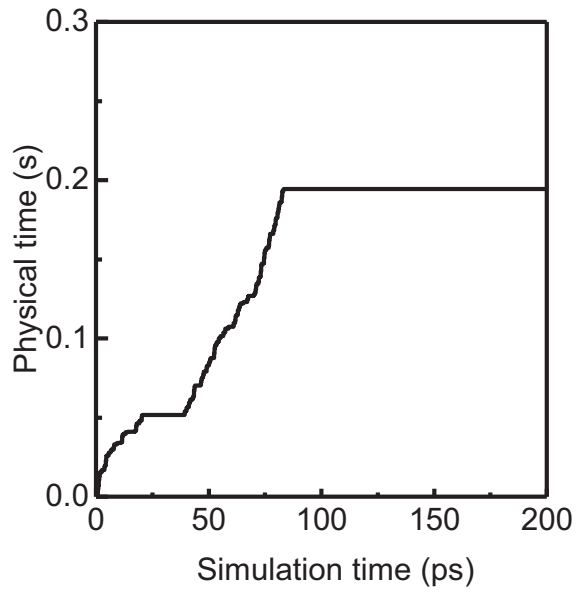

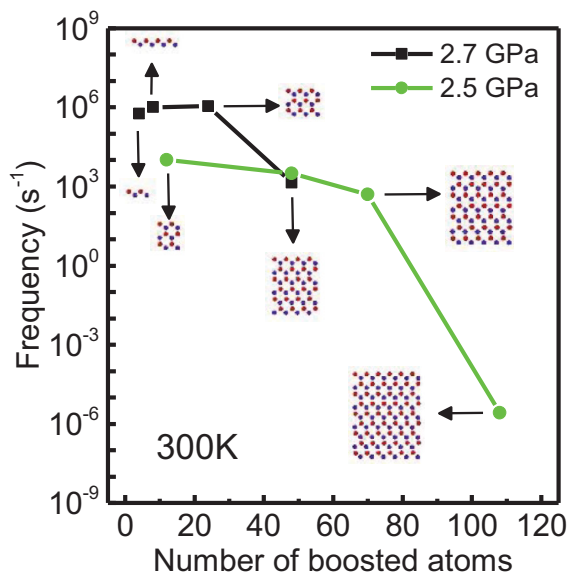

FIG. 2. Relationship between the dislocation-nucleation frequency and the choice of the number of boosted atoms. Insets show the configuration of boosted atoms. Red and blue atoms represent boosted atoms in two adjacent (111) planes.

critical size of dislocation nuclei, the pathway of the nucleation process deviates obviously from that with minimum energy

(b)

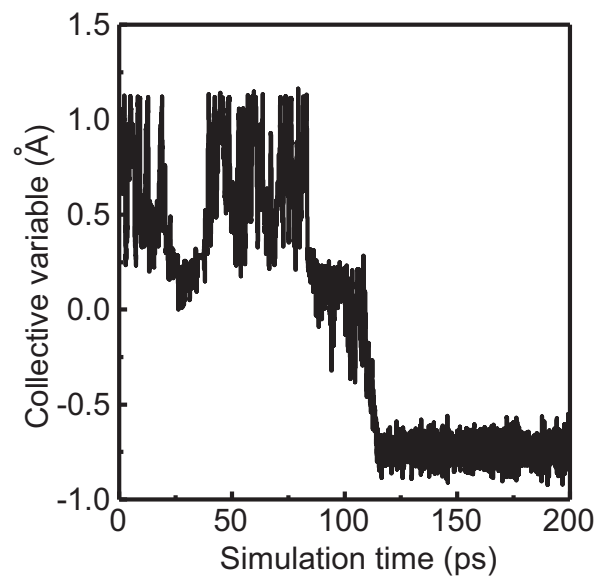

(d)

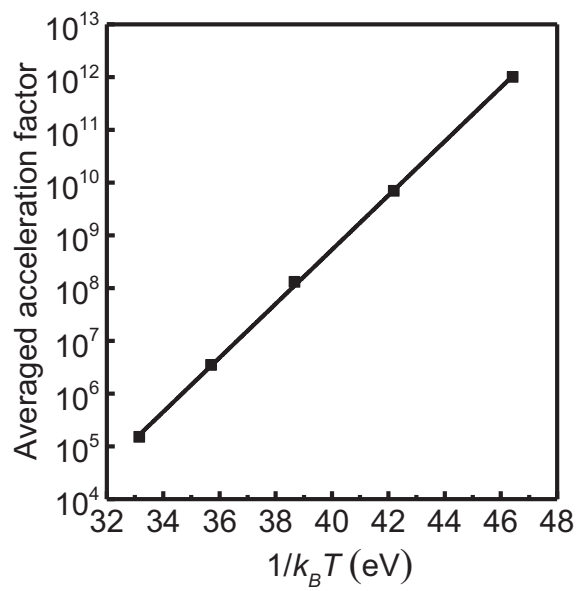

FIG. 3. (a) Typical example of the total boost potential $\Delta V_{\text {total }}^{L}$ constructed after the $L$ th accelerated MD run. (b) Time evolution of CV with respect to simulation time. (c) Accumulated physical time with respect to simulation time. (d) Average acceleration factor at 2.5 GPa and different temperatures. 
(or the critical nuclei). Thus, ABMD with large $N_{\mathrm{b}}$ leads to a pathway with a low nucleation frequency. The number of boosted atoms is carefully selected for ABMD calculation according to the results in Fig. 2.

\section{RESULT AND DISCUSSION}

In the ABMD simulation, the total boost potential constructed after the $L$ th ABMD step is $\Delta V_{\text {total }}^{L}=\sum_{l=1}^{L} \Delta V_{l}$, where $\Delta V_{l}$ is the constructed boost potential in the $l$ th ABMD step using Eq. (1). The total boost potentials at $2.5 \mathrm{GPa}$ and $300 \mathrm{~K}$ are shown in Fig. 3(a) as a typical example. The one-dimensional $\mathrm{CV}$ first oscillates around the initial value $(0.75 \AA)$, and the boost potential $\Delta V_{1}$ is constructed. Then, the oscillation amplitude increases with increasing height of the periodic updated total boost potentials. Finally, the $\mathrm{CV}$ suddenly moves to a new value, which corresponds to a displacement of $1 / 6\langle 112\rangle a_{0}$, where $a_{0}$ is the lattice parameter of face-centered-cubic $\mathrm{Cu}$. A partial dislocation and stacking fault are emitted from the GB. The time evolution of the CV during dislocation nucleation at $300 \mathrm{~K}$ and $2.5 \mathrm{GPa}$ is shown in Fig. 3(b). The physical time $\Delta t$ of each step of regular MD sampling is defined as $\Delta t=\Delta t^{s} e^{\Delta V_{\text {total }}^{L} / k_{\mathrm{B}} T}$, where $\Delta t^{s}=1 \mathrm{fs}$ is the time step, and $\Delta V_{\text {total }}^{L}(L=7$ in the eighth ABMD step in this case) is the immediate value of the total boost potential [33]. The accumulated physical time corresponding to Fig. 3(b) is shown in Fig. 3(c). To give the average nucleation time $(\overline{\Delta t})$ or frequency $(1 / \overline{\Delta t})$, we use the boost potential $\left(\Delta V_{\text {total }}^{L}\right.$, $L=7)$ to calculate the physical time of 11 samples with different initial atomic velocities. The nucleation frequencies converge as the number of samples increases. The acceleration factor, which is the ratio between the average physical time and average simulation time, is shown in Fig. 3(d) for different temperatures at $2.5 \mathrm{GPa}$. The acceleration factor increases exponentially with decreasing temperature.

A conventional MD simulation at $300 \mathrm{~K}$ and $2.8 \mathrm{GPa}$ showed that the $\Sigma 9$ GB emits partial dislocations by the collective multiple dislocation-nucleation mechanism on a time scale of picoseconds (see Figs. 4(a) and 4(b), and Supplemental Movie M_col.wmv [32]). However, at lower uniaxial tensile stress, such as at $300 \mathrm{~K}$ and $2.5 \mathrm{GPa}$, where only accelerated MD can be used, shuffling-assisted single dislocation nucleation appeared first on a time scale of seconds (see Figs. 4(c) and 4(d), and Supplemental Movie M_shuff.wmv [32]). This result suggests that the shufflingassisted single dislocation-nucleation mechanism has a lower free energy than the collective multiple dislocation-nucleation mechanism at lower stress. The activation free energies of the various dislocation-nucleation mechanisms are critical to understanding which dislocation-nucleation mechanism should occur first. To verify the transition between the two dislocation-nucleation mechanisms, we calculate the stress dependence of the activation energy of the mechanisms using the FENEB method [28,29]. Figure 5 shows the results for the activation energy at $0 \mathrm{~K}$. Shuffling-assisted single dislocation nucleation is energetically preferred for $\sigma<3.7 \mathrm{GPa}$, which is about $90 \%$ of the ideal dislocation-nucleation stress (athermal stress) at 4.05 GPa, whereas collective multiple dislocation nucleation is preferred for $\sigma>3.7 \mathrm{GPa}$. Thus, we should use the ABMD method to accelerate shuffling-assisted single dis- (a)

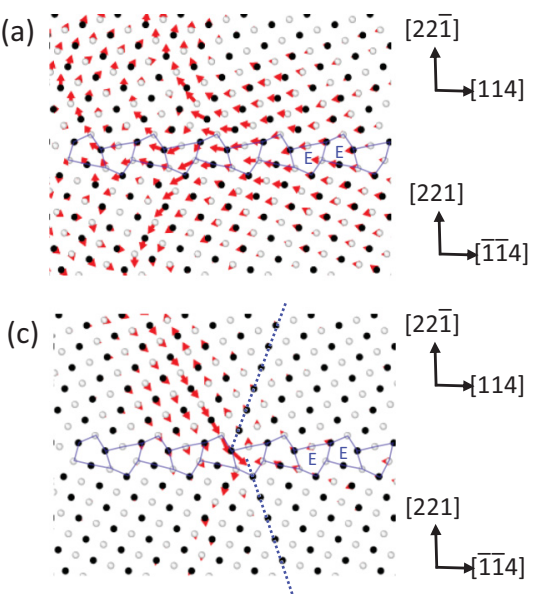

(b)

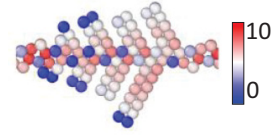

(d)

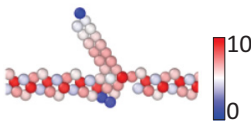

FIG. 4. (a) Atomistic displacement vector field of collective multiple dislocation-nucleation mechanism and (b) corresponding partial dislocation colored according to centrosymmetry [34]. (c) Atomistic displacement vector field of shuffling-assisted single dislocation-nucleation mechanism and (d) corresponding partial dislocation colored according to centrosymmetry. Two dashed lines in (c) label the atom arrays on the upper and lower grains, showing that a shuffling atom shifts from the upper to the lower grain. In $(\mathrm{a}, \mathrm{c})$, black and white atoms denote different (11̄0) atomic planes. Red arrows denote the displacement vectors with respect to the final configurations. For clarity, the magnitudes of the displacement vectors are doubled.

location nucleation instead of collective multiple dislocation nucleation at low stress. To calculate the activation free energy $Q(\sigma, T)$ at finite temperature, the nucleation frequency $v(\sigma, T)$ given by the ABMD or conventional MD is related to $Q(\sigma, T)$ by

$$
v(\sigma, T)=N v_{0} \exp \left[-\frac{Q(\sigma, T)}{k_{\mathrm{B}} T}\right],
$$

where $v_{0} \approx 10^{11} \mathrm{~s}^{-1}$ is the attempt frequency, which is calculated from the curvature of the minimum energy pathway [18], $N$ is the number of equivalent nucleation sites, and $k_{\mathrm{B}}$ is the Boltzmann constant. For the conventional MD at high stress, $v(\sigma, T)$ is calculated directly from the average dislocation-nucleation frequency of 20 samples with different initial velocities of atoms. $N$ and $v_{0}$ in conventional MD are approximated as the same value as in shuffling-assisted single dislocation nucleation in the ABMD calculations. The nucleation frequencies converge as the number of samples increases in the conventional MD calculations. The activation free energies in the ABMD and conventional MD are also shown in Fig. 5(a). For clarity, Fig. 5(b) shows the enlarged activation free energy at the finite temperatures. The crossover of the activation free-energy-stress plots of the shuffling-assisted single dislocation nucleation and the collective multiple dislocation nucleation still exist at finite temperatures. $Q(\sigma, T)$ decreases dramatically with increasing $T$ at a specific $\sigma$. At $0 \mathrm{~K}$, the activation free energy is as high as $2.25 \mathrm{eV}$ at $2.8 \mathrm{GPa}$. In contrast, the activation free energies at 250 and $300 \mathrm{~K}$ decrease to 0.54 and $0.23 \mathrm{eV}$, respectively, at the same stress of $2.8 \mathrm{GPa}$. The activation free 
(a)

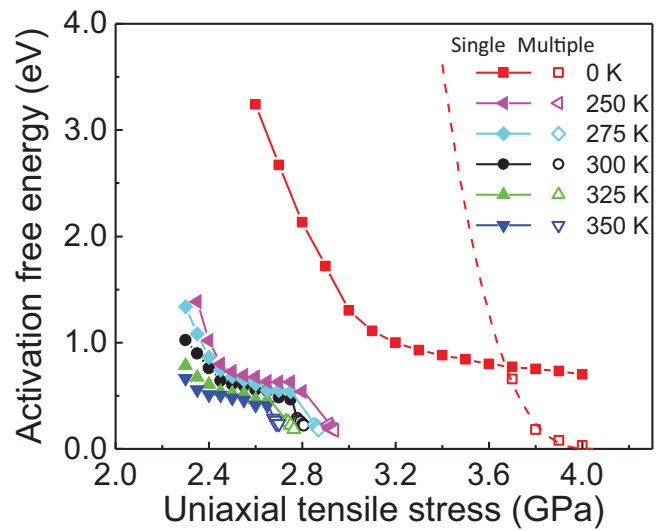

(b)

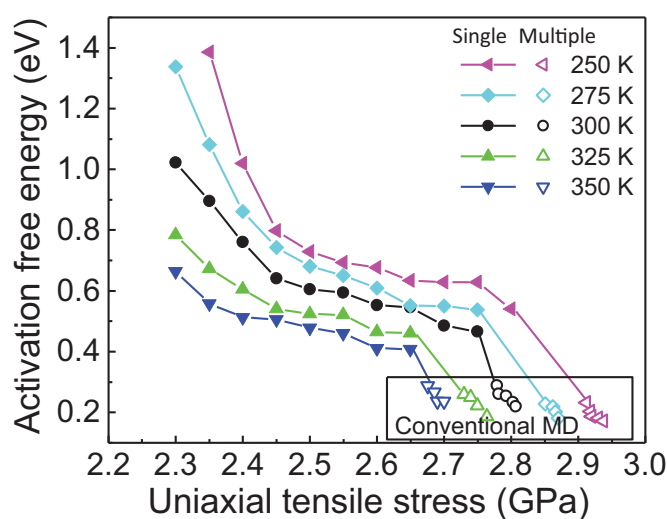

FIG. 5. (a) Activation free energies for shuffling-assisted single dislocation nucleation (solid symbols) and collective multiple dislocation nucleation (open symbols). At $0 \mathrm{~K}$, the activation free energy is calculated by the FENEB method. The dashed line is the fitting result for the activation free energy of collective multiple dislocation nucleation at $0 \mathrm{~K}$ using [18] $Q(\sigma)=A\left(1-\sigma / \sigma_{0}\right)^{\alpha}$, where $A=658.2 \mathrm{eV}$, $\sigma_{0}=4.05 \mathrm{GPa}$, and $\alpha=2.84$. At finite temperatures, the activation free energies are calculated using ABMD (solid symbols) and conventional MD (open symbols). (b) Activation free energies at the finite temperatures for clarity.

energy drops by $90 \%$ as the temperature increases from $0 \mathrm{~K}$ to room temperature. This significant difference in activation free energies demonstrates a strong temperature dependence in the partial dislocation-nucleation process.

The activation volume $\Omega$, which is defined here as $\Omega=$ $-\frac{\partial Q(\sigma, T)}{\partial \sigma}$ with respect to the uniaxial tensile stress, is typically used as a kinetic signature of deformation mechanisms [35]. For example, $\Omega$ is typically on the order of $1000 b^{3}$ for dislocation cutting through forest dislocations in coarsegrained metals, whereas $\Omega$ is between $b^{3}$ and $10 b^{3}$ for surface dislocation nucleation, where $b$ is the magnitude of the Burgers vector. Figure 6(a) shows the $\Omega$ value of the GB serving as a dislocation source according to the FENEB method, conventional MD, and ABMD. The calculated values of $\Omega$ are consistent with the experimental results for nanocrystalline face-centered-cubic metals, such as nanocrystalline $\mathrm{Cu}$ and $\mathrm{Ni}$, where $\Omega$ is on the order of $10-100 b^{3}$ when the grain size is in the range of $20 \mathrm{~nm}$ and hundreds of nanometers [35]. $\Omega$ decreases with increasing stress for either shuffling-assisted single dislocation nucleation or collective multiple dislocation nucleation at $0 \mathrm{~K}$ [see Fig. 6(a)]. At finite temperature, $\Omega$ of shuffling-assisted single dislocation nucleation decreases with increasing temperature. Note that the stress dependence of $\Omega$ of the collective multiple dislocation nucleation at finite temperature is not shown in Fig. 6(a) because of small stress intervals between the data, which cause a large error of $\Omega$. So the average value of $\Omega$ is used in this case. A remarkable characteristic of shuffling-assisted single dislocation nucleation is that $\Omega$ can decrease almost to zero (about $5 b^{3}$, where $b$ is the magnitude of the Burgers vector of a $\langle 110\rangle / 2$ dislocation), well below the athermal stress, in both the FENEB and ABMD calculations. This small $\Omega$ means that the stress cannot effectively decrease the activation free energy further (weak stress dependence); however, the temperature plays the dominant role in activating dislocation nucleation. With increasing stress, $\Omega$ increases dramatically at the stress at which collective multiple dislocation nucleation is energetically favored over shuffling-assisted single dislocation nucleation. The dislocation-nucleation process becomes strongly coupled with the stress, and the (a)

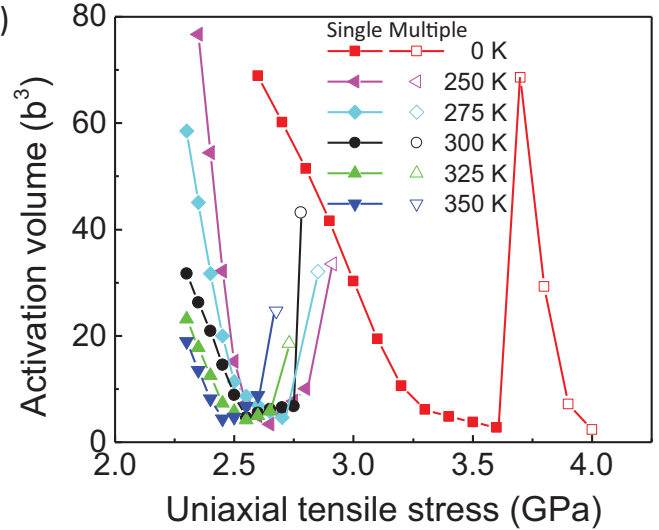

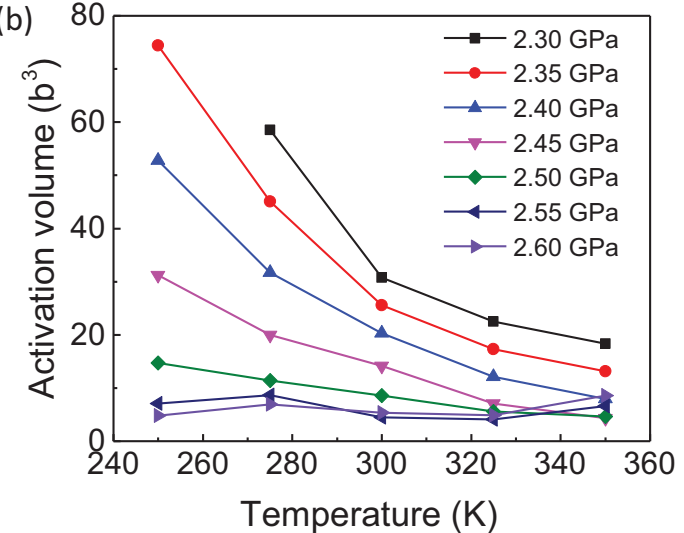

FIG. 6. (a) Activation volume as a function of uniaxial tensile stress for shuffling-assisted single dislocation nucleation (solid symbols) and collective multiple dislocation nucleation (open symbols; at finite temperature, the average value of the activation volume is shown because of the limited available stress interval in conventional MD). (b) Activation volume as a function of temperature for shuffling-assisted single dislocation nucleation. 
activation free energy can be decreased by increasing the stress effectively. Because of the strong stress dependence, the nucleation process of collective multiple dislocation is more "athermal" than that of shuffling-assisted single dislocation. Thus, the effect of the shuffling process on the dislocationnucleation ability of GBs can be discovered only in simulations using accelerated MD. The temperature dependence of $\Omega$ for shuffling-assisted single dislocation nucleation decreases with increasing temperature [see Fig. 6(b)], consistent with experiments on ultrafine-grain materials [36-38]. In the nucleation process in this study, the partial dislocation bows out from the GB, which is in agreement with the thermal activation mechanism suggested by the experiments of ultrafine-grain $\mathrm{Cu}[36,37]$.

Other than the nucleation mechanisms, such as the shuffling-assisted single dislocation nucleation and the collective multiple dislocation nucleation that we have found using ABMD and FENEB, two more single dislocation-nucleation mechanisms are still possible because corresponding to the three inequivalent nucleation sites within a structural unit period of " $E E$," three single dislocation-nucleation modes are expected, as shown in Figs. 7(a)-7(c). According to the dislocation configurations, the nucleation modes in Figs. 7(a) and 7(b) correspond to the shuffling-assisted single dislocationnucleation and nonshuffling single dislocation-nucleation mechanisms, respectively. The dislocation-nucleation mode in Fig. 7(c) is energetically unstable, because the initial dislocation configuration lying in the upper grain spontaneously transforms to a shuffling-assisted dislocation lying in the lower grain. Collective multiple dislocation nucleation from conventional MD near the athermal stress [Fig. 7(d)] can be viewed as being composed of a few nonshuffling single dislocations. The stress dependence of the activation energies of the possible dislocation-nucleation mechanisms in the $\Sigma 9$ GB are calculated using the FENEB method [Fig. 7(e)]. The nonshuffling single dislocation-nucleation mechanism can spontaneously change to the collective multiple dislocationnucleation mechanism in the FENEB method with increasing stress. Note that the activation energy for shuffling-assisted single dislocation nucleation is much larger than 0 near the athermal stress $(\sim 4.05 \mathrm{GPa})$, because the stress dependence (weak coupling with the tensile stress) of shuffling-assisted single dislocation nucleation differs from that of nonshuffling dislocation nucleation. The difference between the shufflingassisted and the nonshuffling dislocation-nucleation mechanisms can be also shown from the saddle configurations given by the FENEB method. For shuffling-assisted single dislocation nucleation [Fig. 7(a)], the structural unit has collapsed at the saddle configurations, while for the nonshuffling single [Fig. 7(b)] or multiple [Fig. 7(d)] dislocation-nucleation mechanisms, the structural unit is only a bit stretched at the saddle configuration (see Supplemental Material [32], Fig. S2). To study whether the loading condition is crucial to crossover of the activation free-energy-stress plots, we also applied complex loading conditions, both tensile and shear loading, on the model and calculated the activation free energy at $0 \mathrm{~K}$ (see Supplemental Material [32], Fig. S3). The result shows that crossover of the activation free-energy-stress plots with increasing stress can still be found. Competition between the dislocation-nucleation modes is associated with the activation volume. Shuffling-assisted single dislocation nucleation has a lower activation energy and smaller activation volume than the other dislocation-nucleation mechanisms at low stress. The lower activation volume corresponds to slow decay of the activation energy with increasing stress. Therefore, at a certain stress, shuffling-assisted single dislocation nucleation will become energetically unfavorable compared with the other mechanisms with larger activation volumes.

The sensitive temperature dependence of the activation free energy in Fig. 5(a) arises from the entropic effect [39-41]. The activation entropy, $\Delta S_{\mathrm{ac}}$, at $300 \mathrm{~K}$ and $2.4 \mathrm{GPa}$ is about $60 k_{\mathrm{B}}$ (see Supplemental Material [32], Fig. S4), which contributes a
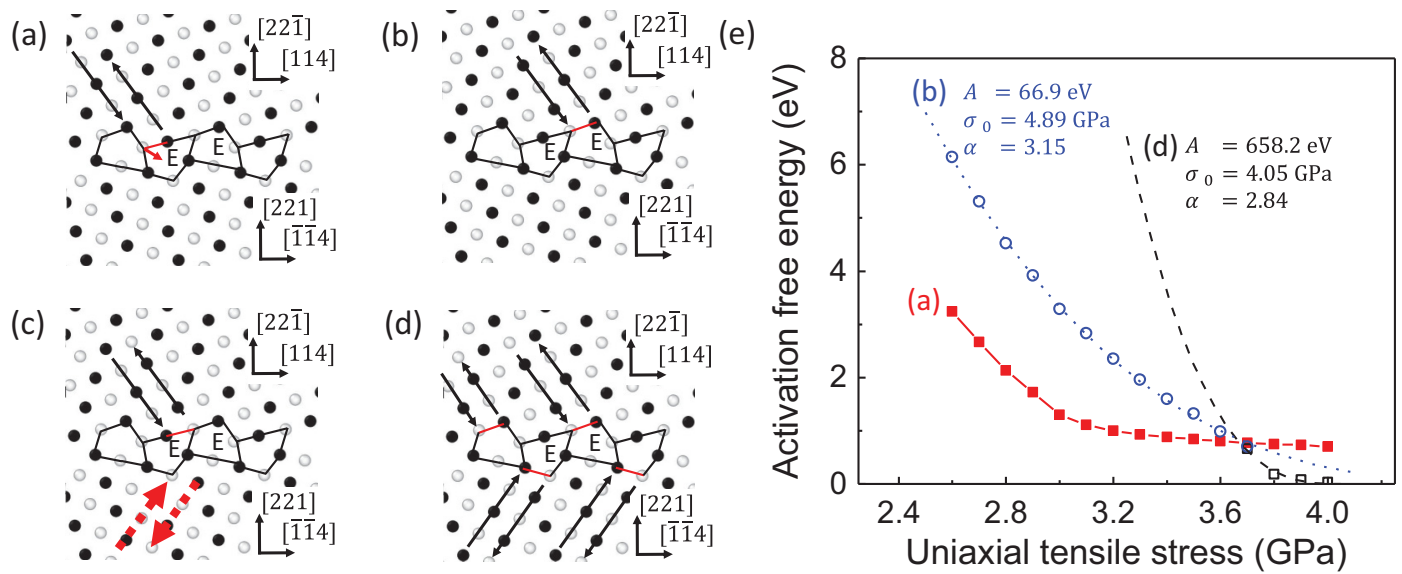

FIG. 7. Competition of dislocation-nucleation mechanisms. (a) Shuffling-assisted single dislocation-nucleation mechanism. Red arrows denote the movement direction of shuffling atoms. (b) Nonshuffling single dislocation-nucleation mechanism. (c) Single dislocation-nucleation mode that is energetically unstable and spontaneously transforms to a shuffling-assisted single dislocation-nucleation mechanism (red, dashed arrows) in FENEB calculation. (d) Collective multiple dislocation-nucleation mechanism given by conventional MD. In (a-d), black and white atoms denote different (1 10$)$ atomic planes. Black arrows denote the relative glide directions of atoms during dislocation emission. Red lines highlight nucleation sites. (e) Activation free energy calculated using FENEB for dislocation-nucleation mechanisms in (a,b,d). Dashed and dotted lines are fitting results using [18] $Q(\sigma)=A\left(1-\sigma / \sigma_{0}\right)^{\alpha}$. 
multiplicative factor $[42,43], \exp \left(\Delta S_{\mathrm{ac}} / k_{\mathrm{B}}\right) \approx 10^{26}$, to the absolute dislocation-nucleation rate. The dislocation-nucleation behavior of imperfect GBs is important to the generality of this study, because flat GBs are rare in realistic cases. We calculated the activation parameters of the second nucleated dislocation from intrinsic stacking fault facets on the GB where an initial dislocation has been emitted (see Supplemental Materials [32], Fig. S5). The result shows that the activation free energy of dislocation nucleation from the imperfect GB is also sensitive to temperature.

In experiments, the test is usually done at constant strain rate, so we calculate the strain-rate dependence of the dislocation-nucleation stress from the GB. For the shuffling-assisted single dislocation nucleation and the collective multiple dislocation-nucleation mechanisms, the critical dislocation-nucleation stress at specific tensile strain rate can be obtained by resolving the equation $[18,44]$

$$
\frac{\mathrm{Q}(\sigma, T)}{k_{\mathrm{B}} T}=\ln \left[\frac{k_{\mathrm{B}} T N v_{0}}{E \dot{\varepsilon} \Omega(\sigma, T)}\right] \text {, }
$$

where $\dot{\varepsilon}$ is the strain rate, $E=\frac{\mathrm{d} \sigma}{\mathrm{d} \varepsilon}$ is the apparent Young's modulus, $v_{0} \approx 10^{11} \mathrm{~s}^{-1}$ is the attempt frequency, and $N=1536$ is the number of equivalent nucleation sites. For simplification, the activation energy $Q(\sigma, T)$ in Fig. 5 is fitted using analytical functions for the two mechanisms (see Supplemental Materials [32]), and activation volume is calculated from the fitting results of activation energy. At a specific strain rate, the mechanism with lower critical nucleation stress should be viewed as the dominating mechanism. The strain-rate dependence of the critical nucleation stress is shown in Fig. 8. From Fig. 8, the shuffling-assisted single dislocation-nucleation mechanism occurs at low strain rate (for example, at $10^{-3} \mathrm{~s}^{-1}$ in the examined temperature range), while the collective multiple dislocation mechanism occurs at high strain rate (for example, at $10^{9} \mathrm{~s}^{-1}$ in the examined temperature range), which has been found in the conventional high-strain-rate MD simulations [25]. The dislocation-nucleation mechanism transition can be found with increasing strain rate, which might have been missed in the conventional MD simulations, and more importantly, it should be observable in actual experiments at experimentally feasible strain rate and temperature, such as at $\sim 10^{1} \mathrm{~s}^{-1}$ and $275 \mathrm{~K}$.

\section{CONCLUSION}

In conclusion, the sensitive temperature dependence of dislocation nucleation from GBs has been uncovered using atomistic simulations. The sensitive temperature dependence

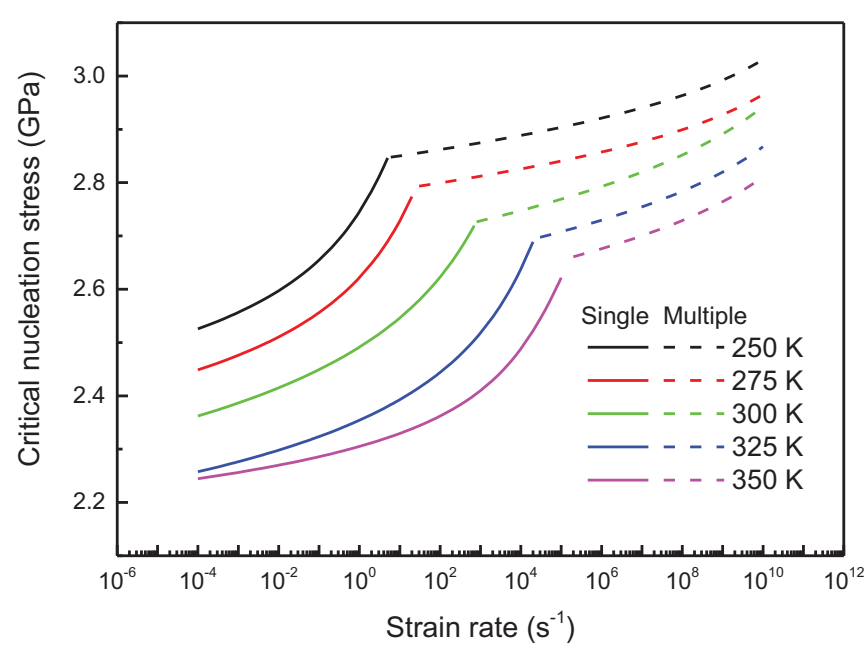

FIG. 8. Strain-rate dependence of the critical nucleation stress for the shuffling-assisted single dislocation-nucleation (solid lines), and that for the multiple collective dislocation-nucleation (dashed lines) mechanisms.

of the activation free energy arises from a large activation entropy, which may be general in the dislocation nucleation from GBs which have the $E$ structural unit. Dislocation nucleation assisted by atomic shuffling is preferred at lower stress, whereas nonshuffling dislocation nucleation dominates the emission process at higher stress. At constant strain-rate condition, the mechanisms' transition from the shufflingassisted dislocation-nucleation mechanism to the multiple collective dislocation mechanism occurs with strain rate increasing from $10^{-4} \mathrm{~s}^{-1}$ to $10^{10} \mathrm{~s}^{-1}$. The shuffling-assisted single dislocation-nucleation mode is not limited to the $\Sigma 9$ STGB. The $\langle 110\rangle$-tilted STGB with misorientation angles between $109.5^{\circ}$ and $180^{\circ}$ and general GBs also contain the $E$ structural unit $[25,26]$, which suggests that these GBs also emit dislocations by atomic shuffling. The strength-structure relationship of GBs [25] should be revisited from the viewpoint of temperature and strain-rate dependence by the methods used in the present study, which combines the accelerated MD, conventional MD, and FENEB methods.

\section{ACKNOWLEDGMENTS}

This work was supported by Grants-in-Aid for Scientific Research in Innovative Area (Grant No. 22102003), Scientific Research (A) (Grant No. 23246025), Challenging Exploratory Research (Grant No. 25630013), and the Elements Strategy Initiative for Structural Materials (ESISM). Y.W. acknowledges financial support from the NSFC (Grants No. 11402269 and No. 11672299).
[1] Z. W. Shan, E. A. Stach, J. M. K. Wiezorek, J. A. Knapp, D. M. Follstaedt, and S. X. Mao, Science 305, 654 (2004).

[2] X. Li, Y. Wei, L. Lu, K. Lu, and H. Gao, Nature 464, 877 (2010).
[3] N. Lu, K. Du, L. Lu, and H. Q. Ye, Nat. Commun. 6, 7648 (2015).

[4] L.Y. Chen, M. He, J. Shin, G. Richter, and D.S. Gianola, Nat. Mater. 14, 707 (2015). 
[5] M. A. Meyers, A. Mishra, and D. J. Benson, Prog. Mater. Sci. 51, 427 (2006).

[6] Z. Shen, R. H. Wagoner, and W. A. T. Clark, Acta Metall. 36, 3231 (1988).

[7] J. Song and W. A. Curtin, Acta Mater. 59, 1557 (2011).

[8] J. R. Rice, in Effect of Hydrogen on Behavior of Materials, edited byA. W. Thompson and I. M. Bernstein (Metallurgical Society of AIME, New York, 1976), pp. 455-466.

[9] A. H. King, Scr. Mater. 62, 889 (2010).

[10] H. Van Swygenhoven, Science 296, 66 (2002).

[11] V. Yamakov, D. Wolf, S. R. Phillpot, A. K. Mukherjee, and H. Gleiter, Nat. Mater. 3, 43 (2004).

[12] J. Schiøtz and K. W. Jacobsen, Science 301, 1357 (2003).

[13] X. Z. Liao, F. Zhou, E. J. Lavernia, S. G. Srinivasan, M. I. Baskes, D. W. He, and Y. T. Zhu, Appl. Phys. Lett. 83, 632 (2003).

[14] H. Van Swygenhoven and J. R. Weertman, Mater. Today 9, 24 (2006).

[15] L. D. Nguyen, K. L. Baker, and D. H. Warner, Phys. Rev. B 84, 024118 (2011).

[16] A. Ishii, S. Ogata, H. Kimizuka, and J. Li, Phys. Rev. B 85, 064303 (2012).

[17] A. Ishii, J. Li, and S. Ogata, PLoS One 8, e60586 (2013).

[18] T. Zhu, J. Li, A. Samanta, A. Leach, and K. Gall, Phys. Rev. Lett. 100, 025502 (2008).

[19] H. Van Swygenhoven, P. M. Derlet, and A. Hasnaoui, Phys. Rev. B 66, 024101 (2002).

[20] M. A. Tschopp, D. E. Spearot, and D. L. McDowell, Dislocat. Solids 14, 43 (2008).

[21] F. Sansoz and J. F. Molinari, Scr. Mater. 50, 1283 (2004).

[22] G. J. Tucker, M. A. Tschopp, and D. L. McDowell, Acta Mater. 58, 6464 (2010).

[23] M. A. Tschopp, G. J. Tucker, and D. L. McDowell, Acta Mater. 55, 3959 (2007).

[24] J. R. Hu, S. C. Chang, F. R. Chen, and J. J. Kai, Scr. Mater. 45, 463 (2001).

[25] D. E. Spearot, M. A. Tschopp, K. I. Jacob, and D. L. Mcdowell, Acta Mater. 55, 705 (2007).
[26] D. Farkas, Curr. Opin. Solid State Mater. Sci. 17, 284 (2013).

[27] Y. Mishin, M. J. Mehl, D. A. Papaconstantopoulos, A. F. Voter, and J. D. Kress, Phys. Rev. B 63, 224106 (2001).

[28] G. Henkelman, B. P. Uberuaga, and H. Jónsson, J. Chem. Phys. 113, 9901 (2000).

[29] T. Zhu, J. Li, A. Samanta, H. G. Kim, and S. Suresh, Proc. Natl. Acad. Sci. U. S. A. 104, 3031 (2007).

[30] D. Faken and H. Jónsson, Comput. Mater. Sci. 2, 279 (1994).

[31] A. Stukowski, Model. Simul. Mater. Sci. Eng. 18, 015012 (2010).

[32] See Supplemental Material http://link.aps.org/supplemental/ 10.1103/PhysRevB.94.104110 for details of calculation of the number of equivalent nucleation site, the structural unit at saddle configuration, the calculation procedure of the activation parameters, dislocation nucleation from imperfect GB or at complex loading, fitting results of the activation free-energystress data, and the accompanying movies.

[33] A. Voter, Phys. Rev. Lett. 78, 3908 (1997).

[34] C. L. Kelchner, S. J. Plimpton, and J. C. Hamilton, Phys. Rev. B 58, 11085 (1998).

[35] R. J. Asaro and S. Suresh, Acta Mater. 53, 3369 (2005).

[36] T. Kunimine, T. Aragaki, T. Fujii, S. Onaka, and M. Kato, J. Mater. Sci. 46, 4302 (2011).

[37] M. Kato, Mater. Sci. Eng., A 516, 276 (2009).

[38] Y. M. Wang, A. V. Hamza, and E. Ma, Acta Mater. 54, 2715 (2006).

[39] S. Ryu, K. Kang, and W. Cai, Proc. Natl. Acad. Sci. U. S. A. 108, 5174 (2011).

[40] Y. J. Wang, A. Ishii, and S. Ogata, Acta Mater. 61, 3866 (2013).

[41] S. Hara and J. Li, Phys. Rev. B 82, 184114 (2010).

[42] A. Yelon, B. Movaghar, and H. M. Branz, Phys. Rev. B 46, 12244 (1992).

[43] A. Yelon, B. Movaghar, and R. S. Crandall, Rep. Prog. Phys. 69, 1145 (2006).

[44] C. R. Weinberger, A. T. Jennings, K. Kang, and J. R. Greer, J. Mech. Phys. Solids 60, 84 (2012). 